\title{
ASPECTOS BIOECOLÓGICOS DE CHELYMORPHA VARIANS BLANCHARD (COLEOPTERA: CHRYSOMELIDAE, CASSIDINAE) DEFOLIADOR DE CONVOLVULÁCEAS
}

\author{
BIOECOLOGICAL ASPECTS OF CHELYMORPHA VARIANS BLANCHARD \\ (COLEOPTERA: CHRYSOMELIDAE, CASSIDINAE), A CONVOLVULACEAE \\ DEFOLIATOR
}

Verónica Cecilia Hamity ${ }^{1}$; Lilia Estela Neder de Román ${ }^{2}$

\begin{abstract}
RESUMEN

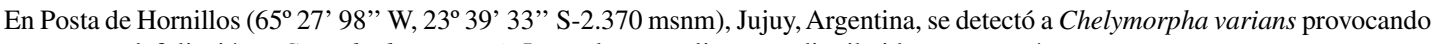
una severa defoliación a Convolvulus arvensis L., maleza ampliamente distribuida en este país.

El objetivo de este trabajo fue conocer aspectos bioecológicos de C. varians (duración del ciclo de vida, mortalidad, comportamiento reproductivo, enemigos naturales) a fin de evaluar su accionar como biocontrolador de esta maleza .

Se realizó el ciclo de vida en laboratorio bajo las siguientes condiciones: temperatura: $25 \pm 1{ }^{\circ} \mathrm{C}$, humedad relativa: $83 \pm 4 \%$ y fotoperiodo normal. La duración promedio en días de los distintos estados y estadios fue: Huevo: 7,25 $\pm 0,4 ;$ Larva: 16,42 $\pm 1,2$ (Larva

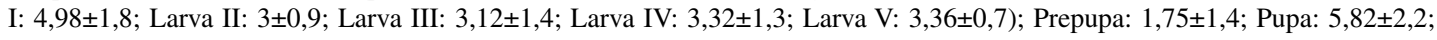
Adultos: 33,37 . El tiempo requerido para la maduración sexual fue de 10 días. Una vez producida la cópula transcurrieron diez días hasta la primera ovipostura. Las mismas fueron realizadas, con intervalos de 2 o 4 días, durante un mes. Las hembras colocaron

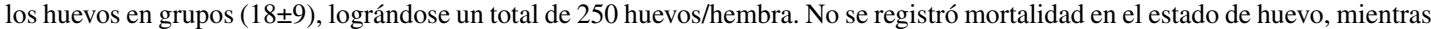
que en los restantes estados inmaduros no alcanzó el $9 \%$.

El único enemigo natural registrado, en campo, fue un microhimenóptero larvipupal (Chalcididae) con un bajo porcentaje de parasitoidismo $(0,7 \%)$.

La facilidad de la cría en laboratorio, el corto ciclo de vida, el alto potencial reproductivo, la mortalidad y el parasitoidismo bajos, son datos básicos cuando se plantean estrategias de manejo de esta importante maleza.
\end{abstract}

Palabras clave: Chelymorpha varians, bioecología, Convolvulus arvensis, manejo

\begin{abstract}

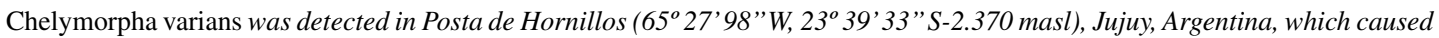
severe defoliation to Convolvulus arvensis $L$., a widely spread weed in this country.

The object of the present work was to study bioecological aspects of $\mathrm{C}$. varians (life cycle, mortality, reproductive behavior, natural enemies) in order to assess its activity as a biocontroller of this weed.

Its life cycle was analyzed in the laboratory under the following conditions: temperature: $25 \pm 1{ }^{\circ} \mathrm{C}$, relative humidity: $83 \pm 4 \%$, and normal photoperiod. Average l+ife duration in days of the different stages and instars was as follows: Egg: 7.25 $\pm 0.4 ;$ Lar-

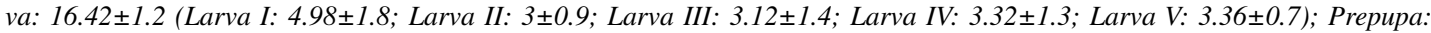

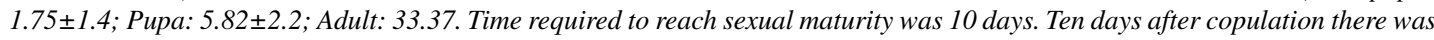
a first egg laying. Then there followed other layings with 2- to 4-day intervals for a month. Female specimens laid eggs in groups (18 99$)$, gathering a total of 250 eggs/female. No mortality was recorded in the egg stage, while in the remaining immature stages mortality was under $9 \%$.

The only natural enemy recorded was a larvipupal microhymenopteron (Chalcididae) with a low parasitoid rate (0.7\%).

Easy laboratory breeding, a short life cycle, high reproductive potential, and low mortality and parasitoidism constitute basic data in the process of setting management strategies for this important weed.

Key words: Chelymorpha varians, bioecology, Convolvulus arvensis, management.
\end{abstract}

1 Instituto de Biología de la Altura, Universidad Nacional de Jujuy. Av. Bolivia 1661. 4600. S. S. de Jujuy; E-mail: vchamity@ inbial.unju.edu.ar.

2 Instituto de Biología de la Altura, Universidad Nacional de Jujuy; Inv. Adj. - CONICET; E-mail: leneder@inbial.unju.edu.ar

Fecha de Recepción: 29 Diciembre 2006

Fecha de Aceptación: 10 Marzo 2007 


\section{INTRODUCCIÓN}

Chelymorpha varians Blanchard es un defoliador especialista que, tanto en estado de larva como adulto, se alimenta de plantas de la familia Convolvulaceae (González, 1989; Artigas, 1994; Suárez y Gianoli, 2006).

Convolvulus arvensis L., "correhuela” o "campanilla", es una especie perenne, voluble y rizomatosa que ha invadido tierras dedicadas a la agricultura en casi todas las regiones templadas del mundo. En Argentina fue declarada plaga de la agricultura por un decreto de 1946 (Rouquaud de Monetti, 1992). Según O’Donell (1959) está presente, especialmente desde Córdoba al sur, donde infesta trigales. En el NOA se la cita sólo para la provincia de Catamarca (Rouquaud de Monetti, 1992).

En la Quebrada de Humahuaca, provincia de Jujuy, Argentina, se detectó a $C$. varians provocando defoliaciones a $C$. arvensis, llamando la atención la abundancia y el importante daño que este crisomélido causaba a las plantas.

El accionar de esta maleza podría afectar la productividad agrícola de la Quebrada de Humahuaca que ha dejado de ser de subsistencia para responder a una economía de mercado nacional e internacional.

El presente trabajo tiene como objetivo conocer aspectos bioecológicos de C. varians (preferencia alimentaria, duración del ciclo de vida, características del adulto, comportamiento reproductivo, mortalidad, enemigos naturales) a fin de evaluar su accionar como potencial biocontrolador de $C$. arvensis en esta zona con características especiales de aridez y altura.

\section{MATERIALES Y MÉTODOS}

El estudio se inició en noviembre de 2005 en la localidad de Posta de Hornillos (65 $27^{\circ} 98^{\prime}$ ' W, $23^{\circ} 39^{\prime} 33^{\prime \prime} \mathrm{S}-2.370 \mathrm{msnm}$.), con la recolección de 140 larvas de los últimos estadios de $C$. varians. En laboratorio fueron colocadas en jaulas de cría, de madera y tela metálica de 38 × 38 × $38 \mathrm{~cm}$, hasta lograr la emergencia de los adultos. A fin de determinar la preferencia y encontrar sustratos alimenticios alternativos, a estos adultos se le ofrecieron tres especies de convolvuláceas, individualizadas en floreros: C. arvensis L., Ipomoea purpurea (L.) Roth e I. batatas (L.) Poir. Con respecto a las larvas se sepa- raron tres lotes de diferentes estados de desarrollo (pequeñas, medianas y grandes). A cada lote de 20 individuos, se le ofreció las mismas especies de convolvuláceas que a los adultos. Para la elección de las mismas se tuvo en cuenta: a) su presencia en la Quebrada de Humahuaca (C. arvensis e I. purpurea) y b) la importancia comercial (I. batatas).

El ciclo de vida se realizó a partir de huevos colocados en laboratorio. Producida la eclosión, se individualizaron 4 lotes de 15 larvas recién nacidas en tubos de farol bajo las siguientes condiciones: $\mathrm{T}^{\mathrm{o}} 25 \pm 1{ }^{\circ} \mathrm{C}, \mathrm{HR} 83 \pm 4 \%$ y fotoperíodo normal. Como sustrato alimenticio se utilizaron, hojas de I. purpurea colocadas en floreros.

Durante el desarrollo de las experiencias se tomaron los siguientes datos: preferencia por el sustrato de alimentación, duración en días de los distintos estados y estadios inmaduros, longevidad y características cromáticas de los adultos, comportamiento reproductivo (tiempo requerido para la maduración sexual y comienzo de la oviposición, ritmo de ovipostura, tamaño de la ovipostura, número total de huevos colocados por hembra), mortalidad en los diferentes estados y estadios y presencia de parasitoides (en el material proveniente del campo).

\section{RESULTADOS Y DISCUSIÓN}

\section{PREFERENCIA ALIMENTARIA}

De las tres especies de convolvuláceas ofrecidas, C. arvensis $\mathrm{I}$. purpurea fueron las aceptadas como alimento por las larvas y adultos de $C$. varians. Se registró una marcada preferencia por la primera en concordancia con lo establecido por González (1989) y Artigas (1994). Con respecto a I. batatas, se observó que no fue apetecible para $C$. varians. Teniendo en cuenta estos resultados y la facilidad para obtener I. purpurea debido a su abundancia en las predios cercanos al laboratorio se eligió a esta especie como sustrato de alimentación para la realización de los ciclos de vida.

\section{CICLO DE VIDA}

La duración promedio en días, bajo condiciones controladas de laboratorio, de los diferentes estados y estadios inmaduros de $C$. varians se muestra en la Figura 1. Del tiempo requerido por esta especie 


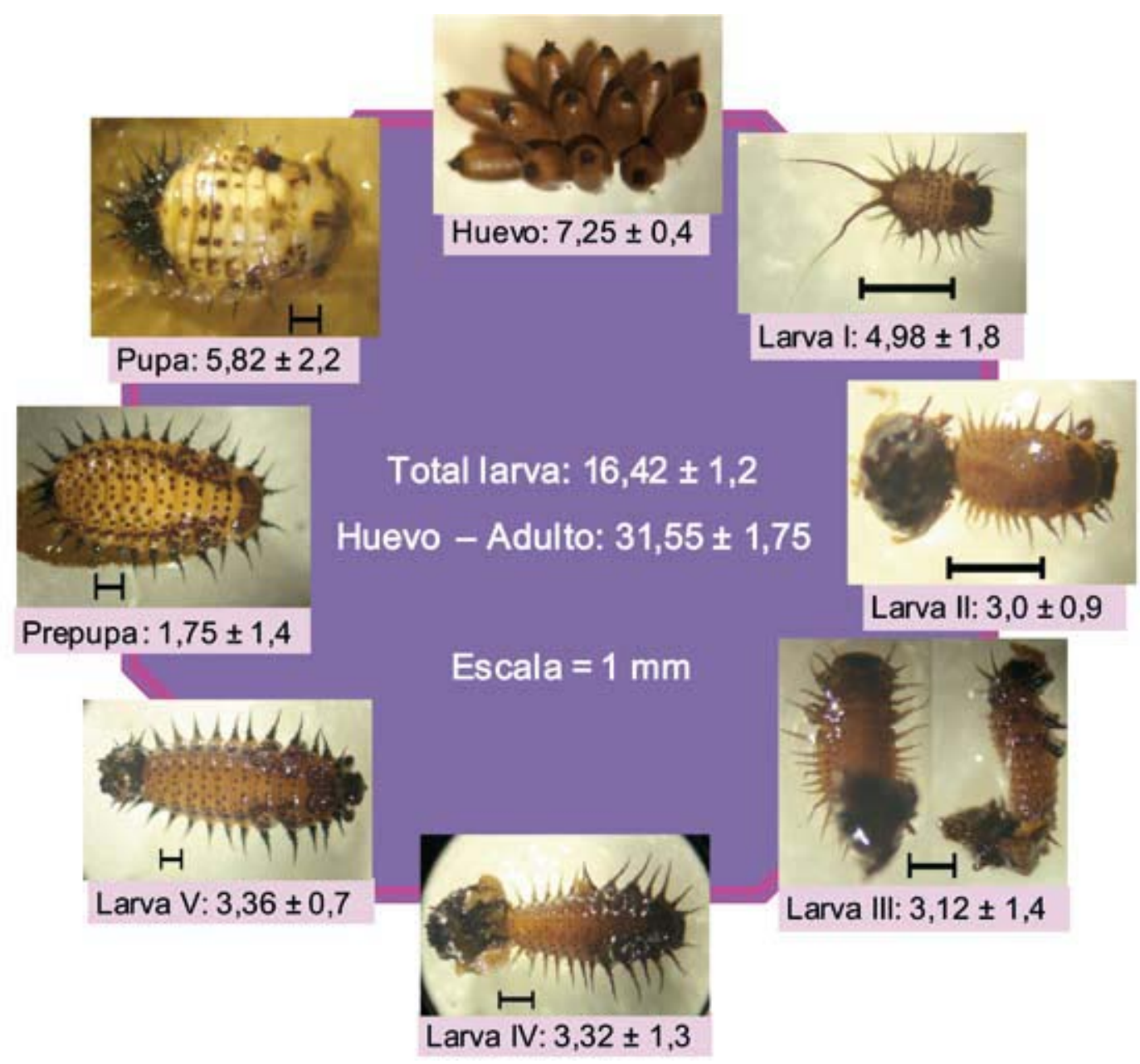

Figura 1. Duración promedio en días de los estados y estadios inmaduros de Chelymorpha varians en condiciones de laboratorio.

para su desarrollo de huevo a adulto $(31,55 \pm 1,75$ días) aproximadamente, la mitad se corresponde con el estado de larva.

La longevidad de los adultos fue variable. El rango en días fue de 2 a 87, siendo el promedio de 33,37 días. Si se considera que una hembra requiere 20 días para comenzar la oviposición, se puede considerar a este valor como el tiempo necesario para que una hembra deje descendencia.

\section{VARIACIONES CROMÁTICAS DEL ADULTO}

Del material recolectado en campo y de las crías obtenidas en laboratorio emergieron adultos con variaciones cromáticas. Las mismas se muestran en la Figura 2 y se podrían corresponder con las formas claras de esta especie propuestas por Borowiec y

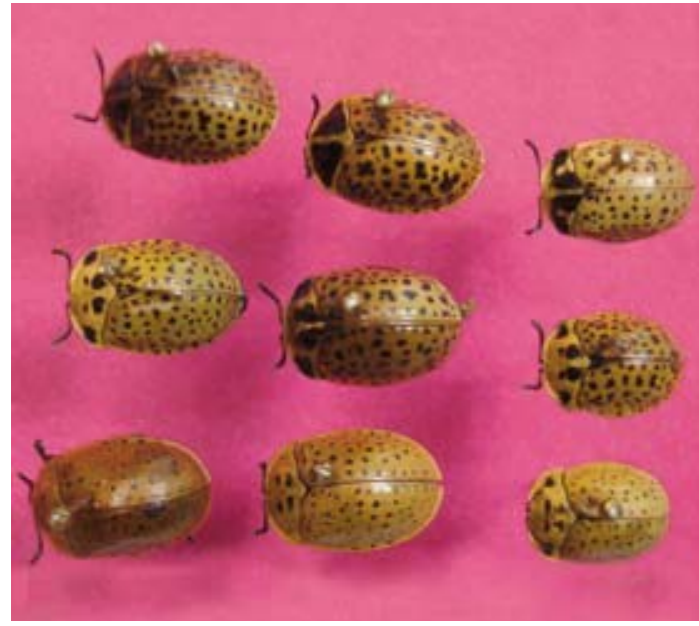

Figura 2. Variación cromática en adultos de Chelymorpha varians. 


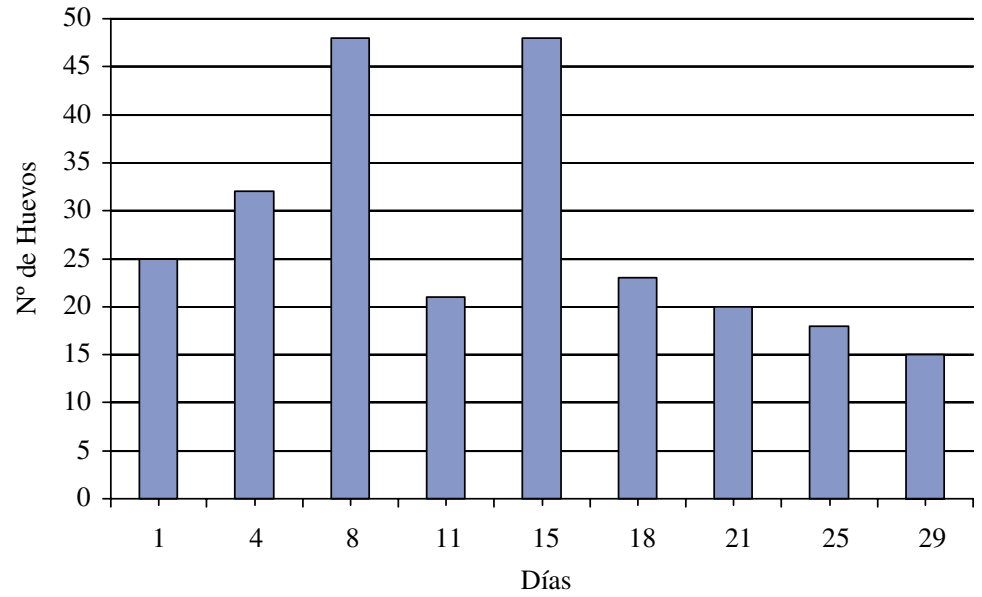

Figura 3. Ritmo de postura y número de huevos colocados por una hembra de Chelymorpha varians.

Swietojanska (2006), ampliándose el número de morfos de 2 a 9. En el estado larval la coloración es uniforme, por lo cual no se pueden inferir las características cromáticas de los adultos.

\section{COMPORTAMIENTO REPRODUCTIVO}

El tiempo previo al apareo fue de 10 días. Posiblemente es el tiempo necesario para lograr la maduración sexual. Una vez producida la cópula, la hembra requirió de 10 días para iniciar la oviposición. Los huevos fueron colocados en grupos (18 \pm 9$)$ con intervalos entre posturas de 2 a 4 días. El número total de huevos depositados por una hembra fue de 250. El ritmo de postura y el número de huevos colocados, en cada una de ellas, se muestran en la Figura 3.

\section{MORTALIDAD-ENEMIGOS NATURALES}

No se registró mortalidad en el estado de huevo. Sólo se constató un bajo porcentaje de mortalidad natural en los estados de larva y pupa $(<9 \%)$.

De las 140 larvas recolectadas en campo, sólo se obtuvo un parasitoide larvi-pupal solitario (Figura 4), que pertenece a la familia Chalcididae, cuyo accionar es escaso no superando el $1 \%$ de parasitoidismo. En Chile, Olivares Donoso et al. (2000) encuentran como parasitodes de huevos de C. varians a Emersonella rotunda (Ashmead) (Hymenoptera: Eulophidae: Entedontinae) y de larvas a Eucelatoria parkeri (Sabrosky) (Diptera: Tachinidae: Goniinae) con un $80 \%$ o más de mortalidad para ambos estados. Es necesario la realización de muestreos específicos en la zona para detectar la existencia de enemigos naturales de los estados de huevo y pupa.

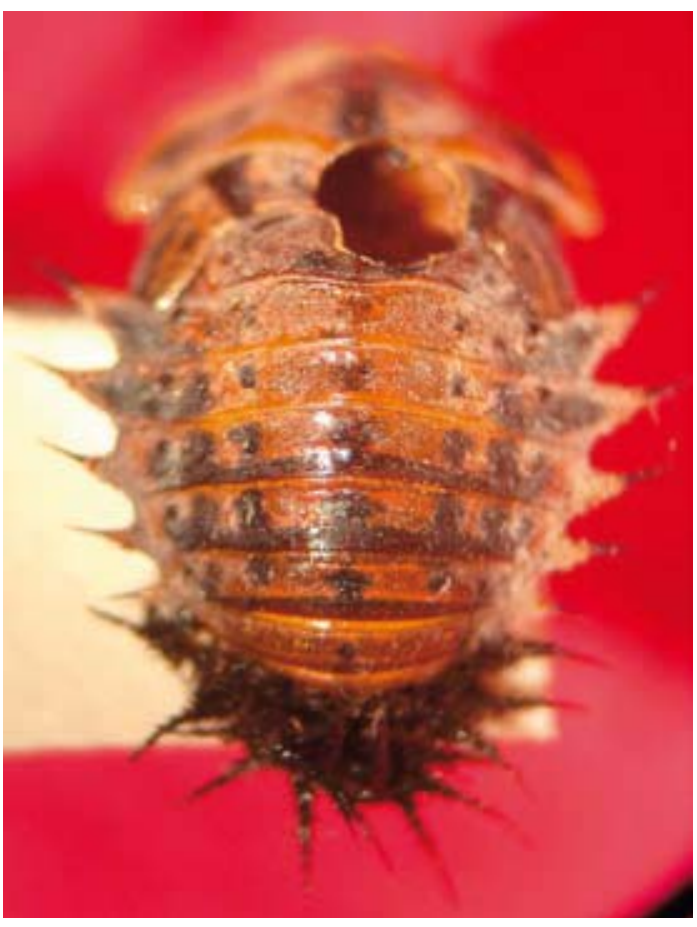

Figura 4. Parasitoide larvipupal (Hym. Chalcididae) de Chelymorpha varians. Perforación realizada en la pupa para su emergencia y adulto.

\section{IMPORTANCIA DE C. VARIANS COMO BIOCONTROLADOR}

C. arvensis es muy difícil de controlar, debido a las reservas considerables de su extenso sistema radical y el poder de regeneración a partir de fragmentos de raíces y rizomas. Es más probable que tenga éxito un programa integrado de manejo de 
malezas, que un programa basado en un solo método (Americanos, 1996).

En Mendoza, Rouquaud de Monetti (1992) establece que el control más efectivo para $C$. arvensis consiste en eliminar la parte aérea de la planta por medio de cortes o utilizando un herbicida. De esta manera se limitan las reservas subterráneas y se impide que la planta semille.

En Jujuy, en la Quebrada de Humahuaca, las labores culturales, indicadas anteriormente se podrían complementar con el accionar de C. varians, dado que esta especie acompaña espacial y temporalmente a $C$. arvensis, la cual incide en los cultivos en las estaciones de mayor productividad agrícola (primavera-verano).

\section{LITERATURA CITADA}

AMERICANOS, P. G. 1996. Malezas de hoja ancha En: Manejo de malezas en países en desarrollo (Estudio FAO: Producción y Protección Vegetal-120). Editores: LABRADA, R., CASELEY J. C. \& PARKER, C. Capítulo 5. Versión online disponible en: http://www.fao.org/docrep/T1147S/ t1147s09.htm

ARTIGAS, J. N. 1994. Entomología Económica. Insectos de interés agrícola, forestal, médico y veterinario. Volumen II. Ediciones Universidad de Concepción. 943 pp.

BOROWIEC, L. \& J. SWIETOJANSKA. 2006. Cassidinae of the world - an interactive manual (Coleoptera: Chrysomelidae). Disponible en: http://www.biol.uni.wroc.pl/cassidae/ katalog\%20internetowy/index.htm

GONZÁLEZ, R. H. 1989. Insectos y ácaros de importancia agrícola y cuarentenaria en Chile. Editorial Ograma. 310 pp.
La facilidad de cría en laboratorio, el alto potencial reproductivo, la mortalidad y parasitoidismo bajos, el corto ciclo de vida, la voracidad de C. varians y la sincronización témporo-espacial con $C$. arvensis son datos básicos a tener en cuenta cuando se plantean estrategias de manejo de esta importante maleza para la zona.

\section{AGRADECIMIENTOS}

Al Ing. Agr. Osvaldo Ahumada (Fac. de Cs. Agr.-UNJu.) por la identificación taxonómica de la especies de Convolvulaceae y al Biólogo Mario Linares (INBIAL-UNJu.) por la realización de las fotos y figura.

O’DONNEL, C. A. 1959. Convolvuláceas argentinas. Lilloa 29: 265-269.

OLIVARES DONOSO, R.; FUENTES-CONTRERAS, E. \& NIEMEYER, H. M. 2000. Identificación de parasitoides de Chelymorpha varians Blanchard (Coleoptera: Chrysomelidae: Cassidinae) en una localidad de Chile Central. Rev. Chilena Ent. 27: 65-69.

ROUQUAUD DE MONETTI, E. 1992. Malezas invasoras de los cultivos mendocinos. Rev. Fac. Cs. Agr. U.N.C. XXV (1-2): 33-46.

SUÁREZ, L. H. \& GIANOLI, E. 2006. Efecto de la herbivoría por un crisomélido especialista sobre la adecuación biológica de la especie nativa Convolvulus demissus (Convolvulaceae). Resumen de la XVII Reunión Anual de la Sociedad Botánica de Chile pág. 4. 